\title{
Maternal obesity legacy: exercise it away!
}

\author{
Petter S. Alm ${ }^{1}$ • Anna Krook ${ }^{1}$ Thais de Castro Barbosa ${ }^{1}$
}

Received: 10 September 2015 / Accepted: 15 September 2015 / Published online: 21 October 2015

(C) Springer-Verlag Berlin Heidelberg 2015

\begin{abstract}
Recent decades have seen maternal obesity during pregnancy increase at an alarming rate. Maternal obesity or extreme weight gain during pregnancy leads to an adverse intrauterine environment that impacts embryonic programming and the offspring's susceptibility to developing chronic metabolic diseases later in life. Regular exercise training is an effective way of postponing or preventing obesity and type 2 diabetes. Maternal exercise training before and during pregnancy has beneficial effects on maternal health and the health of the offspring. Exercise training intervention in obese pregnant mothers attenuates the negative metabolic outcomes in the offspring. But whether exercise training of offspring from obese mothers even later in adult life can counteract these effects is unknown. In this issue of Diabetologia, Bucci and colleagues (DOI:10.1007/s00125-015-3780-8) report that resistance exercise training in elderly women born to obese mothers can improve their metabolic status, overcoming the deleterious impact of maternal obesity. These results underscore that, irrespective of parental legacy, it is never too late to begin an exercise programme and improve your metabolic health.
\end{abstract}

Keywords Maternal obesity $\cdot$ Resistance exercise training · Type 2 diabetes

Obesity and overweight contributes to the worldwide clinical and public health burden, increasing the risk for the development of comorbidities such as insulin resistance, type 2

Thais de Castro Barbosa

thais.de-castro-barbosa@ki.se

1 Department of Physiology and Pharmacology, Karolinska Institutet, von Eulers väg 4a, 17177 Stockholm, Sweden diabetes, frailty and cardiovascular diseases [1-3]. Over the past decade, genome-wide association studies have identified a substantial number of genetic variants associated with the risk for multifactorial forms of obesity and type 2 diabetes, as well as morphological traits such as fat distribution $[4,5]$. However, only a minor portion of the heritability of obesity and type 2 diabetes can be explained by the genetic variants identified to date [6], leaving the mysterious puzzle of the 'missing heritability' unsolved.

More recently, increasing evidence from epidemiological reports and animal studies support an important role for environmental exposure and lifestyle in determining epigenetic marks that may contribute to the heritability of metabolic diseases. These epigenetic marks, defined as DNA methylation, histone modifications and small non-coding RNAs, regulate gene activity without affecting the primary DNA code. Thus, epigenetic processes are strong candidates as mediators of fetal reprogramming, whereby parental nutritional and/or metabolic status regulate the predisposition of the offspring to developing metabolic disorders in later life.

Early evidence for an effect of parental nutritional status on the offspring's susceptibility to metabolic diseases comes from epidemiological data from the Dutch 'Hunger Winter' and the Överkalix studies [7, 8]. These studies linked parental and/or grandparental nutrition during pregnancy or key developmental windows to the children's and grandchildren's risk of developing diabetes, obesity or cardiovascular disease. These observations have subsequently been followed up in a number of clinical and experimental studies demonstrating a relationship between parental obesity/diabetes and low birthweight of the offspring and the subsequent development of metabolic and cardiovascular diseases in adult life. Collectively, these studies implicated an epigenetic basis for the development of these diseases [9]. Particularly, maternal obesity and excessive weight gain during pregnancy showed a strong association 
with childhood obesity and an increased risk for the offspring to develop metabolic diseases in later life. These studies led to the hypothesis that an adverse intrauterine environment affects the fetal metabolic epigenetic programming, and leads to longlasting effects on offspring health and disease [9].

In the current issue of Diabetologia, Bucci and colleagues [10] report on whether maternal obesity affects glucose metabolism of the offspring in later life. Specifically, they investigated whether the deleterious effects of obesity during a period of intrauterine development can be reversed in late adult life by exercise training. Studying a subgroup from the Helsinki Birth Cohort Study (HBSC), the authors explored the metabolic effects of resistance exercise in frail elderly (septuagenarian) women born to either obese or normal weight mothers. The two groups of women were matched for BMI and were normoglycaemic. Consistent with previous observations, women whose mothers had been obese or overweight tended to be more insulin resistant and had impaired skeletal muscle glucose uptake compared with women whose mothers were of normal weight. The study participants then undertook a 4 month resistance exercise intervention, with training sessions three times per week. Studies in humans, as well as in animal models, have shown that maternal exercise training during pregnancy mitigates the negative effects of maternal obesity on the metabolic health of the offspring
[11-13]. Both the mother and fetus benefit from the positive effects of the maternal exercise training [11-13]. However, the question of whether the negative metabolic effects of an unfavourable intrauterine milieu caused by maternal obesity can be improved or reversed in the offspring by exercise training later in adult life has up till now remained unanswered.

The 4 month exercise programme resulted in a small but significant and comparable increase in skeletal muscle mass in elderly women who were born to either a normal weight or obese mother. In the elderly women born to normal weight mothers, resistance exercise had little or no impact on the metabolic outcomes measured. However, skeletal muscle glucose uptake and whole-body insulin sensitivity was improved in elderly women born to obese or overweight mothers. After the training period, glucose uptake and insulin sensitivity in daughters of obese or overweight mothers were comparable to those born to lean mothers. Thus, although the exercise regimen prescribed in this study did not lead to any appreciable improvement in insulin sensitivity in women born to normal weight mothers, the women born to obese mothers showed improved metabolic outcomes (Fig. 1). Furthermore, exercise training reduced the intracellular lipid content in skeletal muscle from women born to obese or overweight mothers. Again, this exercise effect was not evident in daughters born to lean mothers, underscoring the possibility that women born to

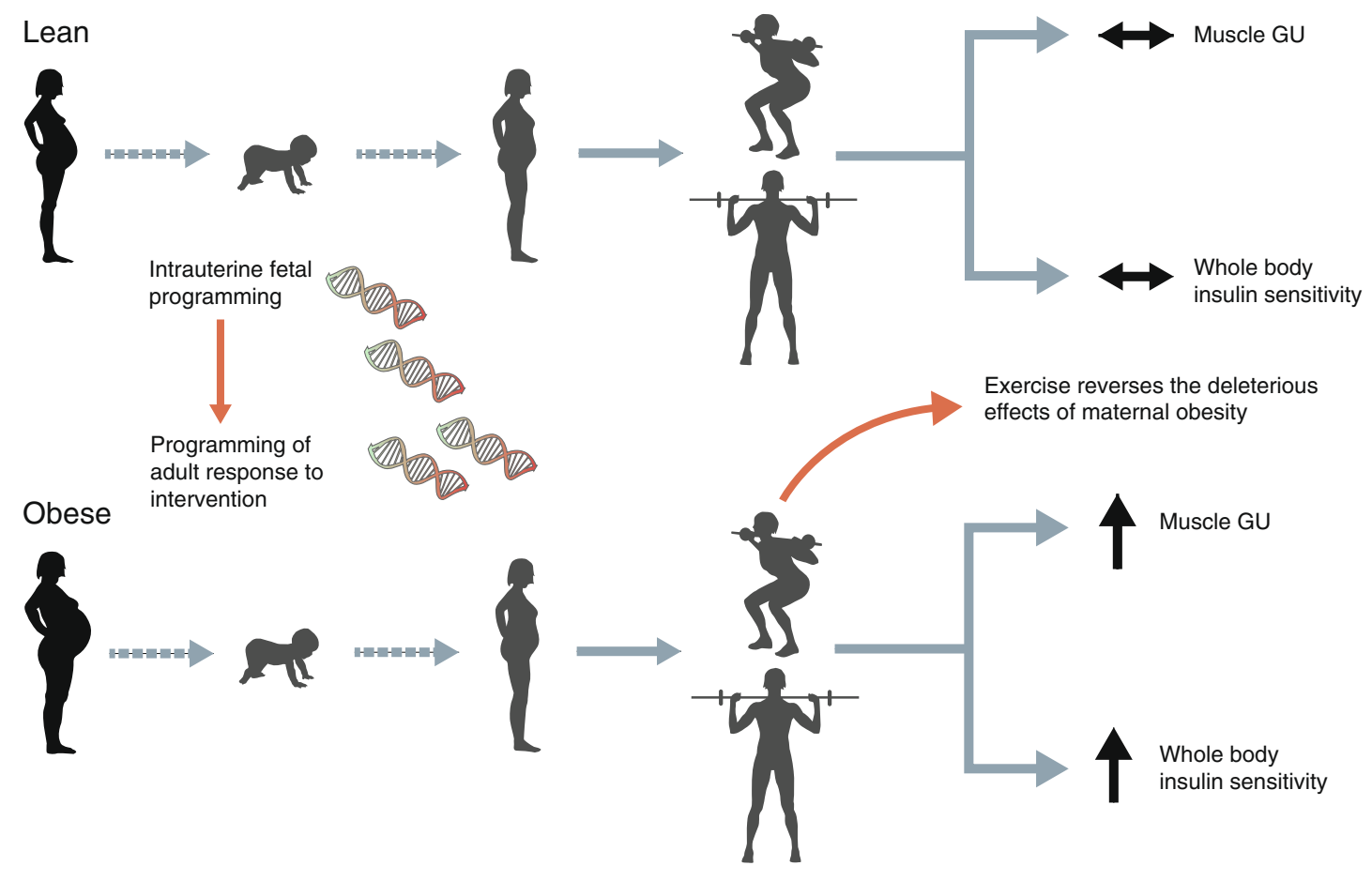

Fig. 1 Resistance training reverses maternal environmentally induced transmission of metabolic disease. The intrauterine milieu plays a central role in epigenetic programming of metabolic diseases in childhood as well as in adulthood. Maternal obesity or excessive weight gain during pregnancy impacts the offspring's predisposition to metabolic disorders, such as obesity and type 2 diabetes (T2D). Resistance exercise training in

adult offspring born to lean mothers does not alter skeletal muscle glucose uptake (GU) or whole-body insulin sensitivity. However, resistance training in adult offspring from obese mothers improves muscle GU and whole body insulin sensitivity, reversing the negative effects of maternal obesity, thus raising the possibility that maternal obesity during pregnancy impacts the offspring's responsiveness to exercise interventions 
obese mothers may have a more sensitive and/or rapid response to exercise training. Whether this reflects a different epigenetic programming in the skeletal muscle of women born to obese mothers, or whether this is the result of an initial 'less favourable' insulin sensitivity at baseline, irrespective of the cause, is unclear. The authors also determined leucocyte telomere length and found that amongst daughters from obese mothers, those with the shortest telomere lengths at baseline showed the largest improvement in insulin sensitivity following the exercise intervention. This relationship was not evident in daughters born to lean mothers, and since the cohorts were of modest size, additional studies are warranted to further probe the relationship between telomere length and exercise response.

The women selected for exercise intervention in the current study were all frail, although no significant differences in measures of insulin sensitivity were noted when comparing frail and non-frail daughters born to lean mothers. Nevertheless, it remains possible that frailty per se may have an impact on the outcomes measured. Since all the women had normal fasting glucose at age 70 , the study population may also be somewhat skewed if one assumes that more women born to obese mothers would have developed overt type 2 diabetes at this age. Thus, the women included in this study born to obese mothers may be particularly responsive to exercise intervention and/or enriched for genes which are protective with respect to metabolic disease.

Lifestyle intervention, including diet and physical activity are key factors for preventing and treating obesity and type 2 diabetes. The positive effects of exercise training in improving skeletal muscle insulin sensitivity and reducing adiposity is well established. Furthermore, exercise training is an important therapeutic intervention during pregnancy for the attenuation or prevention of adverse health outcomes in both mother and offspring. For example, 5-year-old children of mothers who exercised during pregnancy were much leaner than offspring of sedentary mothers [14]. Experimental studies in mice have reported that exercise training before and during pregnancy improved the detrimental effects of maternal highfat-diet feeding on the glucose tolerance and fat mass of offspring [12]. Epigenetic modification of genes involved in metabolism may be one potential mechanism by which metabolic disorders induced by adverse intrauterine conditions are mediated. In mice, hypermethylation of the promoter of the master metabolic regulator peroxisome proliferator-activated receptor $\gamma$ coactivator- $1 \alpha$ (PGC- $1 \alpha$ ) was prevented by maternal exercise, concomitant with improved metabolism in the adult offspring [11]. Nutritional status and exercise training during pregnancy are thus critical components affecting the development of childhood or chronic adult diseases.

Bucci and colleagues add an important perspective for a role for exercise training in improving glucose metabolism and reveal that lifestyle intervention may override an unfavourable maternal legacy. Indeed, if individuals with the greatest 'risk' for developing metabolic disorders are the most responsive to exercise intervention, this highlights a need for additional studies and tailored personalised intervention programmes. A number of important questions remain to be determined, such as: how persistent or how plastic are the effects of intrauterine programming? To what extent are the deleterious effects of the intrauterine environment on metabolic health reversible? Are certain time points in life more suitable for intervention than others? Does maternal obesity during pregnancy impact the offspring's responsiveness to exercise intervention? What are the key mechanisms mediating these effects, and are they also possible to target using pharmacological intervention?

While these questions will take time to be fully addressed, the data presented in this issue of Diabetologia highlight that your own lifestyle choice has a stronger impact on your health than your parent's lifestyle. Thus, exercise training in adulthood may overcome the negative legacy effects of maternal obesity.

Duality of interest The authors have no conflicts of interest.

Contribution statement The authors were the sole contributors to this article. All authors were responsible for drafting the article and revising it critically for important intellectual content. All authors approved the version to be published.

\section{References}

1. Bouillon K, Kivimäki M, Hamer M et al (2013) Diabetes risk factors, diabetes risk algorithms, and the prediction of future frailty: the Whitehall II prospective cohort study. J Am Med Dir Assoc 14: 851.e1-6

2. Flegal KM, Graubard BI, Williamson DF, Gail MH (2007) Causespecific excess deaths associated with underweight, overweight, and obesity. JAMA 298:2028-2037

3. Kelly T, Yang W, Chen CS, Reynolds K, He J (2008) Global burden of obesity in 2005 and projections to 2030 . Int J Obes (Lond) 32: 1431-1437

4. Heid IM, Jackson AU, Randall JC et al (2010) Meta-analysis identifies 13 new loci associated with waist-hip ratio and reveals sexual dimorphism in the genetic basis of fat distribution. Nat Genet 42: 949-960

5. Speliotes EK, Willer CJ, Berndt SI et al (2010) Association analyses of 249,796 individuals reveal 18 new loci associated with body mass index. Nat Genet 42:937-948

6. McCarthy M (2015) Genomic medicine at the heart of diabetes management. Diabetologia 58:1725-1729

7. Lumey LH, Stein AD, Kahn HS et al (2007) Cohort profile: the Dutch Hunger Winter families study. Int J Epidemiol 36:1196-1204

8. Pembrey ME, Bygren LO, Kaati G et al (2006) Sex-specific, maleline transgenerational responses in humans. Eur J Hum Genet 14: 159-166

9. Rando OJ, Simmons RA (2015) I'm eating for two: parental dietary effects on offspring metabolism. Cell 161:93-105 
10. Bucci M, Huovinen V, Guzzardi MA (2015) Resistance training improves skeletal muscle insulin sensitivity in elderly offspring of obese mothers. Diabetologia doi:10.1007/s00125-015-3780-8

11. Laker RC, Lillard TS, Okutsu M et al (2014) Exercise prevents maternal high-fat diet-induced hypermethylation of the $P g c-1 \alpha$ gene and age-dependent metabolic dysfunction in the offspring. Diabetes 63:1605-1611

12. Stanford KI, Lee MY, Getchell KM, So K, Hirshman MF, Goodyear LJ (2015) Exercise before and during pregnancy prevents the deleterious effects of maternal high-fat feeding on metabolic health of male offspring. Diabetes 64:427-433

13. Mourtakos SP, Tambalis KD, Panagiotakos DB et al (2015) Maternal lifestyle characteristics during pregnancy, and the risk of obesity in the offspring: a study of 5,125 children. BMC Pregnancy Childbirth 15:66

14. Clapp JF 3rd (1996) Morphometric and neurodevelopmental outcome at age five years of the offspring of women who continued to exercise regularly throughout pregnancy. J Pediatr 129:856-863 